\title{
On Fabrication and Mechanical Testing of a New Finger Support
}

\author{
Simin Nasseri*, Vitale Kyle Castellano and Mushfequr Kotwal \\ Mechanical Engineering Department, Kennesaw State University, 1100 S. Marietta Pkwy, Marietta, Ga, 3000, USA
}

Received 05 May 2018, Accepted 08 July 2018, Available online 10 July 2018, Vol.8, No.4 (July/Aug 2018)

\begin{abstract}
Following the work done previously on design and simulation of a new finger support, this paper focuses on mechanical testing, methods of fabrication using a LaserJet $3 D$ printer and also explores a better polymer which can be used for the support. The special composite finger support was designed for the first time to accommodate the patients with all sort of finger deformity. A new model is presented here, which is the optimum design suggested previously and is consisted of polymeric support with an inserted sheet of aluminum or carbon fiber. After presenting the results of modeling (including the calculated maximum stress and maximum deformation), the maximum stress values were compared with the yield strength values of the metals and it was noticed that the support does not undergo any plastic or permanent deformation. Furthermore, in order to validate the modeling results, the support was fabricated and mechanical tests were conducted. The steps and details of designing, simulation, mathematical confirmation using MATLAB (Mathworks@) package, fabrication, and mechanical testing are all presented. Overall the finger support is reliable and strong and can be brought to the market.
\end{abstract}

Keywords: Arthritis, Polymers, Composite, 3D printing, Finite Element Analysis, Modeling.

\section{Introduction}

Finger deformities have many underlying causes, including diseases such as rheumatoid arthritis7, osteoarthritis (Egger, et al, 1995), swan-neck deformity (Giesen, 2009), and so on. In many cases, the finger is bent at the base to a point where it cannot be straightened without a splint, therapy, or surgical and non-surgical methods. A cost-effective way to fix a bent finger may be a finger guide, which the finger inserts into. While wearing the support, the patient will be able to perform daily functions.

There are various finger deformities, with root causes and the effects on fingers which were discussed in detail in the first paper. Mallet finger (Padiachee, 2016), also known as "baseball finger", occurs from injury to the extensor tendon where it attaches to the distal phalanx. The failure in treatment of mallet finger deformity might lead to swan-neck deformity (Giesen, 2009). Dupuyten's contracture (Foucher, et al, 2001) is a genetic disorder that causes thickening and scarring of tissue in the palm, which in turn causes the fingers to bend toward the palm. Boutonniere Deformity (Sameh, et al, 2012) causes hyperextension of the distal interphalangeal or DIP. Clawfinger (Brandsma, 1982) bends the Metacarpal phalangeal joint outward, yet also bends the other joints inward.

*Corresponding Author's Mail snasser1@kennesaw.edu (ORCID ID:0000-0002-6036-053X)

DOI: https://doi.org/10.14741/ijcet/v.8.4.8
The goal of this study is to create a finger support that enables the patient to perform daily functions, while providing comfort and flexibility. The support would help relieve pain during times of inflammation and would allow the finger to straighten over time. Finger supports which are currently on the market, do not accommodate for many things, such as comfort, or multiple finger positions.

Additive manufacturing, interchangeable with 3D printing, is a rapidly growing technology which works by adding layer upon layer to create 3D models (Attaran, 2017), (Giesen, 2009). Baumers et. al. reviewed the energy consumption and cost in 3D printing and designed a method to accurately estimate the build time (Baumers, et al, 2015).

3D printing was chosen as the fabricating method for this study, as it is cheaper and faster than molding and extrusion. At first an elastomer, TangoPlus; a polymer known for its high flexibility, was chosen as the material for 3D fabrication. It was proved that this polymer is not suitable for applications in which the polymer is subjected to extension, bending, etc. Instead DuraForm ${ }^{\circledR}$ Flex was chosen which is more tear resistant and durable.

One primary model was designed, and then replicated with different materials in SolidWorks (C) Simulation. This model was fabricated and tested to ensure the model's reliability and durability. This paper presents the simulation and fabrication results for this model and briefly explains the mechanical 
testing method and suggests the best materials for the support.

\section{Materials and Methods}

For this study, a model was designed that can be worn for both resting (with no applied load) and functional positions (performing normal life tasks). The model comprises of a vertical spine that rests on the bottom of the finger (holding a sheet of Aluminum or Carbon Fiber), and three rings which wrap around the finger. This will provide the support with strength and rigidity and will hold the force of the finger when bending. The finger support has been tapered to follow the shape of a finger. Bevels were created to reduce stress concentrations at specific areas such as internal cavities and the rings. Small cuts were made between the rings to provide bending of the support and to accommodate any bend angle. The finger support allows the finger to "breathe" and allows small deformations without too much fatigue due to stretching (Figure 1).

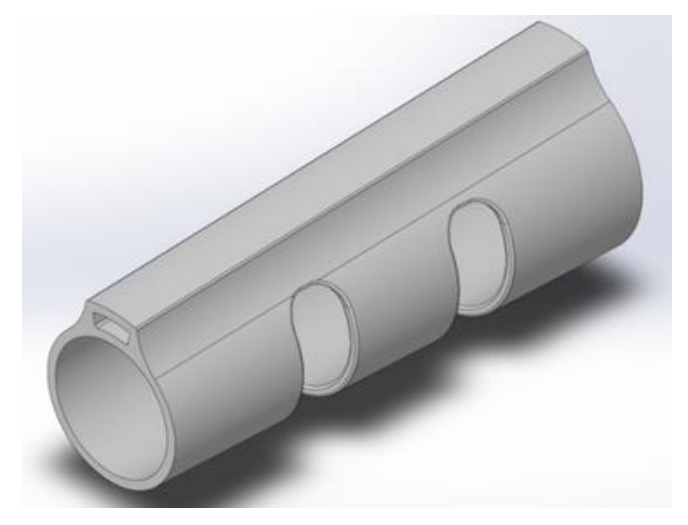

Fig.1 Composite finger support comprised of the flexible polymer and inserted sheet of metal (not shown here). The support accommodates different bend angles and also is tapered to follow the shape of a finger.

The finger's force is applied to the aluminum or carbon fiber sheet, which acts as a thin plate, and can be treated as a cantilever beam (the base bears no force). Thus, the appropriate equations for a cantilever beam could be used to find bending stress, deflections, and moments.

Silva, et. al. conducted a study to determine the forces that act on the joints of a finger by connecting a dynamic splint with a dynamometer. The researchers used trigonometric equations to calculate the flexing force at angles ranging from $25^{\circ}-45^{\circ}$. From the study, it was observed that the larger the finger, the greater the force that is exerted on the tendon (Silva, et al, 2005). Hofmann and Goebl8 explored the finger force profiles and tongue techniques of clarinet students and professional clarinetists under controlled performance conditions. Their study found that the finger force varied between different body types and sex
(Hofmann, 2016). The results yielded an average flexion force of $1.17 \mathrm{~N}$. The largest force of $1.21 \mathrm{~N}$ was recorded during high expression level performance conditions, which is consistent with other researcher's findings who used dynamometers (Silva, et al, 2005). This force is considered for this present work as "the base force."

A finger which is bending can act as a cantilever beam with distributed forces acting along the length of the finger. For a finger exerting a force on one point, the deflection of the aluminum sheet is given by: (1) where $\mathrm{F}$ is the force exerted by the finger, $\mathrm{L}$ is the length of the beam, $\mathrm{E}$ is the elastic modulus of the metal, and I is the mass moment of inertia in mm4. For aluminum 6061-T6, the modulus of elasticity (E) is $69 \mathrm{GPa}$. The moment of inertia (I) for a rectangular cross section is given by (bt3)/12, where $b$ is the width of the strip in $\mathrm{mm}$, and $\mathrm{t}$ is the thickness in $\mathrm{mm}$.

The conjugate beam method is used to derive the displacement and slope of a beam. The length of the conjugate beam is equal to the length of the original beam, but the load on the conjugate beam is given by $\mathrm{M} / \mathrm{EI}$ of the original beam. Hence, the following equations were used to validate the Finite Element Analysis process and results: (2)

$\mathrm{M}$ is the moment produced by each force in $\mathrm{N} \neg-\mathrm{mm}$, and $\mathrm{a}$ is the location of the force on the beam relative to the fixed end in $\mathrm{mm}$.

A MATLAB (Mathworks(C) program was created to calculate the maximum stress and deflection of a metal beam under the applied forces by reading the user's inputs. The program was written to validate the results from the simulation. The code also reported the factor of safety by considering a yield, or tensile, strength of the material versus the calculated stress to estimate how much the forces can be increased 10 .

\subsection{Simulation}

Finite Element Analysis (FEA) was conducted using SolidWorks to evaluate the support with the forces acting on it. The analysis also took into consideration the aluminum sheet metal and the TangoPlus support Bevels were created in certain parts of the model to lower stress concentration in corners. The interior section of the rings were also rounded to avoid tearing when bending. Only the mandatory fillets were used in FEA analysis. It should be noted that the 3D printed prototype was printed with all bevels and rounds.

Three-point loads of $1.21,0.806$, and 0.403 Newtons were applied internally at $51.1175 \mathrm{~mm}$, $35.2425 \mathrm{~mm}$, and $19.3675 \mathrm{~mm}$ from the base of the support, respectively. The support was fixed on one end to represent a cantilever beam.

Meshing complexities led to problems in simulation. While refining the mesh provides results with greater accuracy, refining the mesh too much causes divergence in stress plots. To avoid singularities, area segments using the split line feature had to be created to apply the forces in very small regions. These small 
regions were circular with diameter of 0.010 inches. Additionally the forces were normal to the curved circular areas in which they were respectively applied too.

An H-adaptive method was used to obtain convergence. The $\mathrm{H}$-adaptive method runs multiple studies, and automatically refines the mesh based on the results of the previous study. Figure 2 shows a larger version of the mesh used for clarity purposes. The inset shows the refined mesh.

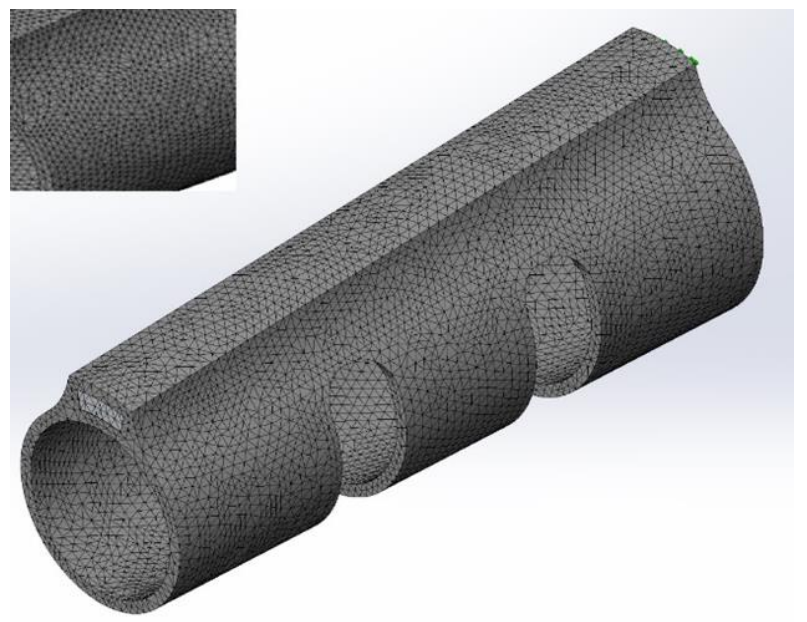

Fig.2 H-adaptive Mesh. An H-adaptive solver was used to automatically refine the mesh and obtain convergence. Please note that the mesh shown is not the one used when running the analysis. This was done for clarity purposes; the inset shows a segment of the actual refined mesh.

The stress and displacement were calculated for four different material combinations (polymer and metal), which had the same dimensions, mesh size, and forces. These FEA studies are summarized in Table 1: FEA Finger Support Material Studies.

Table 1: FEA Finger Support Material Studies.

\begin{tabular}{|c|c|c|}
$\begin{array}{c}\text { Study } \\
\text { Number }\end{array}$ & Vertical Sheet Material & $\begin{array}{c}\text { Finger Support } \\
\text { Material }\end{array}$ \\
\hline $\mathbf{1}$ & Aluminum 6061-T6 (SS) & TangoPlus \\
\hline $\mathbf{2}$ & Carbon Fiber & TangoPlus \\
\hline $\mathbf{3}$ & Aluminum 6061-T6 (SS) & DuraForm ${ }^{\circledR}$ Flex \\
\hline $\mathbf{4}$ & Carbon Fiber & DuraForm ${ }^{\circledR}$ Flex \\
\hline
\end{tabular}

\subsection{Fabrication}

The model was fabricated using a 3D Polyjet printer. The finger support material was chosen to be TangoPlus Fullcure 930, with the resin material being Fullcure 705. Carbon fiber was chosen instead of aluminum to be inserted into the vertical slot (Figure 3). In the previous study, a waterjet was used to remove support material after printing, which caused the finger support to tear. The final support was soaked in a $2 \%$ sodium hydroxide solution for two hours, after which it was rinsed with tap water to remove support resin. In this work, the model was soaked in sodium hydroxide for one hour, with subsequent submergences for 5 minutes to aid in the Fullcure 705 removal process. The best method was to remove the Fullcure 705 by hand and then submerge for 5 minutes, which was performed 3 times.

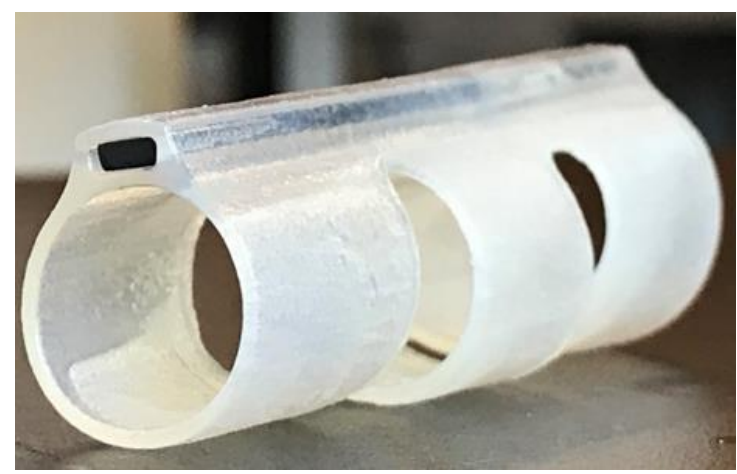

Fig.3 The finger support comprised of a flexible polymer and inserted sheets of Aluminum or carbon fiber

\subsection{Mechanical Testing}

The support was tested after fabrication to verify the results of the simulation. The carbon fiber sheet was inserted into the vertical slot, and the support was worn around the finger of a subject. The finger was clamped to the table to represent a cantilever beam position (Figure 4). Instead of three-point loads along the finger, an equivalent load was attached via a hangar near the tip of the finger. The hangar was centered on top of a dial indicator. Hence, a hanger was designed which would accommodate varying loads.

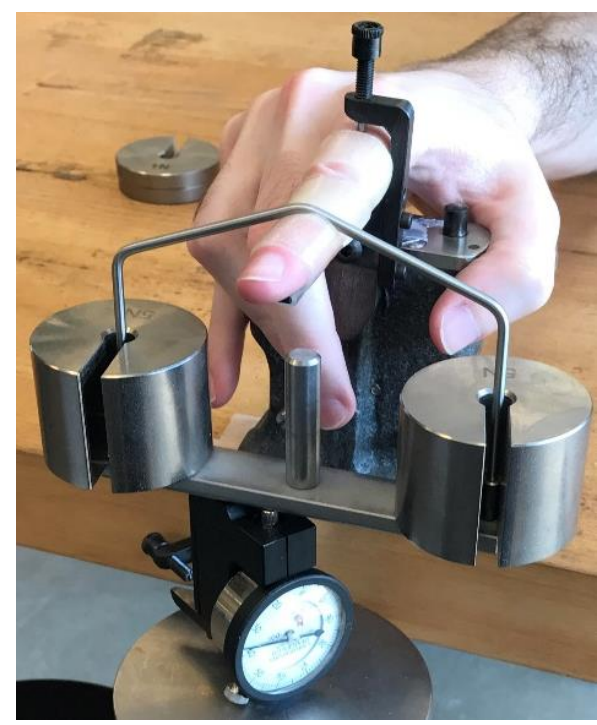

Fig.4 Mechanical testing for validating the strength of the composite finger support which is subjected to various loads

A 0.001 " x 0.250 " dial indicator was used to measure the displacements. The dial indicator included a large 
scale ranging from 0-100 and a small scale ranging from 0-2.5. Each mark on the large scale represents 0.001 inches, while each mark on the small scale represents 0.250 inches. The calibration was first done for the dial. Once the load was applied, the pointer came into contact with the surface of the hanger and hence the plunger dropped into the dial to record the displacements.

The hangar provided the ability to adjust the load. The first test was conducted using a load of $4 \mathrm{~N}$. The load was increased to $10 \mathrm{~N}$ for the second test.

\section{Results}

The model designed in this study helps patients with finger deformity by providing comfort and flexibility, which reduces pain during rest and activity. The design featured a composite support made of three rings, with an aluminum sheet to provide a vertical backbone.

The starting material was chosen to be a rubberlike polymer known as TangoPlus Fullcure 930, which is soft, flexible, and helps decrease irritation. However, the fabrication process shows that this material should not be used for similar applications. Instead more tear resistant polymers, such as the DuraForm $®$ Flex, should be used.

A MATLAB program was written to calculate the stress and displacement of a cantilever beam under the forces applied. The results from the SolidWorks (C) simulation yielded nearly the same values for stress and displacement.

The von Mises theory (Kurowski, 2016) compares the maximum stress obtained with the yield strength of the material used, stating that the material will fail if the applied stress is greater than its yield strength. In the simulation, the maximum stress from the simulation was far below the yield strength of aluminum (or Tensile strength of CF). The H-adaptive method was used to converge the results from various meshes.

The $\mathrm{H}$-adaptive method was used with a maximum mesh of 0.020 in and a minimum mesh of 0.005 in. Solving for nine loops yielded a converged stress result. This $\mathrm{H}$-adaptive mesh was then applied to studies with different beam material and support material identical to those listed in Table 1: FEA Finger Support Material Studies.

For an aluminum 6061-T6 (SS) sheet and TangoPlus the maximum von Mises stress of $53.02 \mathrm{MPa}$ and a maximum deflection of $0.9271 \mathrm{~mm}$ were found. Figure 5 shows the stress and displacement results.

For evaluation purposes, the model was replicated using carbon fiber instead of aluminum inside the vertical slot, and with TangoPlus as the support material. The von Mises stress was found to be 55.69 $\mathrm{MPa}$ (the difference is because in FEA displacement is calculated first, which is then used to find stress results ${ }^{10}$ ) and the displacement was $0.2827 \mathrm{~mm}$.
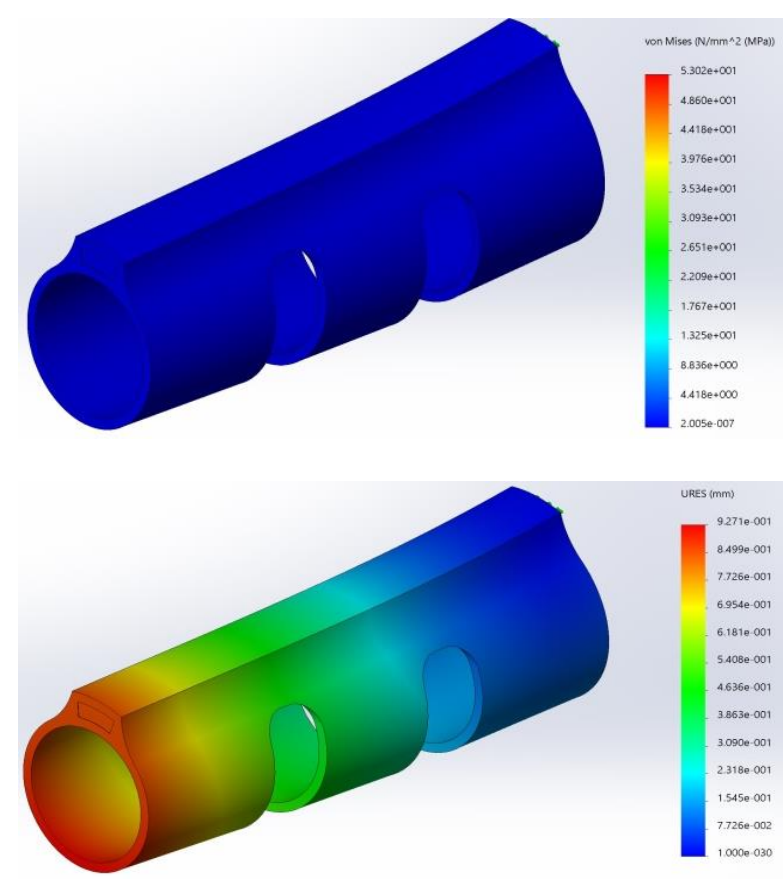

Fig.5 Von-Mises stress values for the finger loads applied (Left or 5A) and displacement throughout the composite support (Right or 5B)

The considerably lower displacement proves that a carbon fiber sheet would provide much higher strength. A feasible alternative to TangoPlus, with superior tear resistance of $15.1 \mathrm{~J} / \mathrm{m}$ was found to be DuraForm $\AA$ Flex which can be 3D printed. With this material simulation results with aluminum 6061-T6 (SS) yielded a von Mises stress value of 52.90 MPa and a displacement of $0.9217 \mathrm{~mm}$. Additionally DuraForm $®$ Flex with carbon fiber yielded a von Mises stress value of $55.66 \mathrm{MPa}$ and $0.2822 \mathrm{~mm}$ displacement. Both of these additional studies are congruent with the TangoPlus, mainly because the forces are mainly tolerated by the vertical sheet material. Nonetheless, a higher tear resistance would be ideal for this concept. Table 2: Stress and Displacement Results, summarizes these values.

Table 2: Stress and Displacement Results.

\begin{tabular}{|c|c|c|}
\hline $\begin{array}{c}\text { Study } \\
\text { Number }\end{array}$ & von Mises Stress (MPa) & $\begin{array}{c}\text { Displacement } \\
\text { (mm) }\end{array}$ \\
\hline $\mathbf{1}$ & 53.02 & 0.9271 \\
\hline $\mathbf{2}$ & 55.69 & 0.2827 \\
\hline $\mathbf{3}$ & 52.90 & 0.9217 \\
\hline $\mathbf{4}$ & 55.66 & 0.2822 \\
\hline
\end{tabular}

In the work conducted in the previous study, using a maximum mesh of $0.03 \mathrm{in}$. and a minimum mesh of about $0.01 \mathrm{in}$. yielded a maximum stress of $45.83 \mathrm{MPa}$ and a maximum deflection of $0.5537 \mathrm{~mm}$. It is important to note that the length of the support in the previous study was 2 in., while the new model is 2.25 in. Also, the previous model was very different in terms of geometry. Thus, it is not feasible to compare the two models in terms of stress and displacement. However, 
the new model is a much more improved design, as it provides a more comfortable fit around the finger and is more resistant to tearing. Comparing these results to the MATLAB code is useful to judge the validity of the FEA analysis. Table 3 tabulates the code's outputs for aluminum 6061-T6 (SS) and carbon fiber.

Table 3: MATLAB Results.

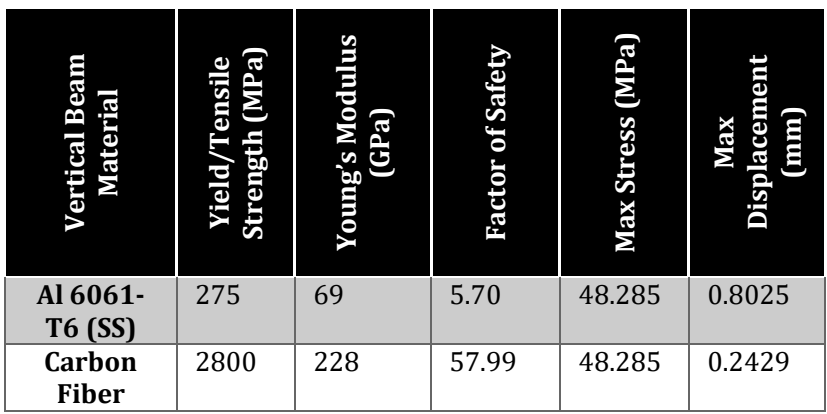

These results are acceptably close. It should be noted that the MATLAB code only considers the beam without the support. Besides, it considers a rectangular beam, not a curved beam that the FEA analysis considers. The maximum stress here is found at the end of the cantilever beam, which is the fixed end. Nonetheless these values and the FEA simulation show that the finger support presented here is valid. Finally the maximum forces are presented in Table 4: Maximum Forces, which is based again on the MATLAB function

Table 4: Maximum Forces that the Support Can Handle

\begin{tabular}{|c|c|c|c|c|}
\hline $\begin{array}{c}\text { Vertical } \\
\text { Beam } \\
\text { Material }\end{array}$ & $\begin{array}{c}\text { Max } \\
\text { Force 1 } \\
\text { (N) }\end{array}$ & $\begin{array}{c}\text { Max } \\
\text { Force 2 } \\
\text { (N) }\end{array}$ & $\begin{array}{c}\text { Max } \\
\text { Force 3 } \\
\text { (N) }\end{array}$ & $\begin{array}{c}\text { Sum } \\
\text { (N) }\end{array}$ \\
\hline $\begin{array}{c}\text { Al6061-T6 } \\
\text { (SS) }\end{array}$ & 6.891 & 4.590 & 2.295 & 13.777 \\
\hline $\begin{array}{c}\text { Carbon } \\
\text { Fiber }\end{array}$ & 70.167 & 46.740 & 23.370 & 140.277 \\
\hline
\end{tabular}

Mechanical testing was done to measure the displacement of the finger support after being attached to equivalent loads representing the maximum force that can be applied to a carbon fiber beam.

These tests were performed after fabrication to validate the results of the simulation and evaluate the overall effectiveness of the support. The finger support was worn by a test subject, and equivalent weights representing a high functional load $(10 \mathrm{~N})$, withstood by a carbon fiber beam, were hung from the finger to measure the displacement.

The deformations were mainly due to the entire finger bending at the base, because the carbon fiber did not bend at all. The test verified that the support works well with the daily functional loads applied on a finger which are usually much less than the maximum load predicated for the composite support with carbon fiber (about $140 \mathrm{~N}$ ). When the finger was completely clamed and fixed, the dial showed no more than few marks, representing a fraction of millimeter of deformation (descending the hanger).

\section{Conclusions and Discussions}

Finger supports for high finger functionality have not been researched heavily in the industry. Thus, this study could advance the current crop of products in the market today. The goal of the study was to design a support that straightens a bent finger overtime, while providing comfort and finger functionality. The support would also help during inflammation and relieve pain during moments of discomfort.

In this work, one model was designed with an inserted metal sheet. The idea was to create a support that is not only comfortable, but strong and able to help patients perform daily functions. The model was replicated to simulate stress and deflection using vertical sheet materials aluminum 6061-T6 (SS) and carbon fiber, as well as different support material TangoPlus and DuraForm $®$ Flex.

The simulation results of this model cannot be compared to the results of the previous study due to the designs having different lengths and different geometries. The higher stress and displacement in the new model could be due to having a longer length than the previous model. The new support improves upon several factors of the previous one, such as lowering stress accumulation in corners, being more resistant to tear, providing various bend angles and a better grip around the finger.

Several changes could be made in the fabrication process. TangoPlus should not be used because of its low tear resistance. Other materials could be chosen for better results, such as TangoBlack, TangoGray, or Agilus 30 FLX, all of which have higher tear resistance than TangoPlus. Even other DuraForm $\AA$ products could be considered.

With access to a higher functioning printer, much thinner supports could be printed. Additionally, shape changing polymer structures could be created by designing multi-material composites, a process also known as 4D printing9. While designing and manufacturing using this approach is currently very difficult, it has great potential for future modeling of complex shapes. For this work, 3D printing was chosen to fabricate the finger support, as it faster and cheaper than other methods.

A study should also be conducted to better understand the relationship between sodium hydroxide and material deterioration. The previous model was soaked in a sodium hydroxide for approximately two hours because it had the resin material in more areas such as the interior rings. The new model only contained the resin material in the vertical slot and externally between the rings, which only required one hour of soaking. A fatigue analysis could increase the understanding of how long the finger support can hold the fluctuating loads of the finger. 
This new model, having gone through simulation, fabrication, and mechanical testing, can be used by patients to help relieve pain caused by finger deformity, and can assist with finger functionality while straightening the finger over time. With further studies conducted on fabrication processes and materials, researchers can explore more complicated models.

\section{References}

M. Attaran, (2017), The rise of 3-D printing: the advantages of additive manufacturing over traditional manufacturing, Business Horizons, Vol.60, No.6, pp. 677-688.

M. Baumers, R. Wildman, C. Tucker, P. Dickens, R. Hague (2015), Modeling build time, process energy consumption and cost of jetting-based additive manufacturing, $N P$ \& Digital Manufacturing Conference, Vol. 2015, No.1, pp. 311316.

J. W. Brandsma, J. M. Watson, (1982), Clawfinger Correction: results of extensor-flexor many tailed operation, The Hand, Vol.14, No.3, pp. 307-12.

P. Egger, C. Cooper, D. J. Hart, D. V. Doyle, D. Coggon, T. D. Spector, (1995), Patterns of joint involvement in osteoarthritis of the hands: the Chingford study, J Rheumatol, Vol.22, No.8, pp. 1509-1513.

G. Foucher, C. Lequeux, J. Medina, D. Nagel, (2001), A congenital hand deformity: dupuytren's disease, Journal of Hand Surgery, Vol.26, No.3, pp. 515-517.

A. Georgescu, I. Capota, I. Matei, (2013), A new surgical treatment for mallet finger deformity: deepithelialised pedicled skin flap technique, Injury, Vol.44, No.3, pp. 351355
F. J. V. D. Giesen, W. J. V. Lankveld, C. Kremers-Selten, A.J. Peeters, E.B. Stern, S. Le Cessie, R. G. H. H. Nelissen and T. P. M. Vliet Vlieland, (2009), Effectiveness of two finger splints for swan neck deformity in patients with rheumatoid arthritis: a Randomized, Crossover trial, Arthritis \& Rheumatism, Vol.61, No.8, pp. 1025-1031.

A. Hofmann, W. Goebl, (2016), Finger forces in clarinet playing, Frontiers in Psychology, Vol.7, No.1140, pp. 1-12.

S. Kantareddy, T. Simpson, Z. Ounaies, M. Frecker. (2016), 3D printing of shape changing polymer structures: design and characterization of materials, Proceedings of the 26th Annual International Solid Freeform Fabrication Symposium - An Additive Manufacturing Conference, pp. 2224-2235.

P. M. Kurowski, (2016), Engineering analysis with SolidWorks Simulation, Mission: SDC Publications, pp. 5-30 \& 211-227 \& 238-281.

A. Lurati, (2017), Dupuytren's Contracture, Workplace Health and Safety, Vol.65. No.3, pp. 96-99.

C. Padiachee, F. Shahid, N. Jamayet, M. Alam, (2016) Management of mallet finger injury by alam prosthetic device (APD), International Medical Journal, Vol.23, No.6, pp. 700-701.

E. Sameh, T. Aly, 0. Amin, M. Hegazi, (2012), Surgical management of chronic boutonniere deformity, Hand Surgery, Vol.17, No.3, pp. 359-364.

S. N. P. da Silva, S. Nicolau, R. Mattar, R. B. Neto, C.A.M Pereira, (2005), Measurement of the flexing force of the fingers by a dynamic Splint with a dynamometer, Clinics, Vol.60, No.5, pp. 381-388.

L. Wang, T. Meydan, P. Williams, (2017), Design and evaluation of a 3D printed optical sensor for monitoring finger flexion, IEEE Sensors Journal, Vol.17, No.6, pp.1937-1944. 